\title{
Sonomicrometry-derived 3-dimensional geometry of the human tricuspid annulus
}

\author{
Marcin Malinowski, MD, ${ }^{\mathrm{a}, \mathrm{b}}$ Tomasz Jazwiec, MD, ${ }^{\mathrm{a}, \mathrm{c}}$ Matthew Goehler, BS, ${ }^{\mathrm{a}}$ Nathan Quay, BS, ${ }^{\mathrm{a}}$ \\ Jared Bush, BS, ${ }^{a}$ Stefan Jovinge, MD, PhD, ${ }^{a}$ Manuel K. Rausch, PhD, ${ }^{d}$ and Tomasz A. Timek, MD, PhD ${ }^{\mathrm{a}}$
}

\section{ABSTRACT}

Objectives: Surgical correction of functional tricuspid regurgitation is focused on prosthetic reduction and remodeling of the tricuspid annulus. We set out to investigate the precise geometry of the human tricuspid annulus to better guide surgical therapy.

Methods: Eleven human donor hearts with normal right ventricular function and without tricuspid regurgitation that were rejected for clinical transplantation were harvested. Sonomicrometry crystals were sewn around the tricuspid annulus and pressure sensors placed in the right ventricle and right atrium. The hearts were studied in the TransMedics Organ Care System (Andover, Mass) ex vivo perfusion apparatus in the right heart working model. Data were acquired at baseline and before and after bolus calcium infusion. Annular height, dimensions, strain, and curvature were calculated based on 3-dimensional crystal coordinates.

Results: Maximal annular area was $997 \pm 258 \mathrm{~mm}^{2}$ and minimal $902 \pm 257 \mathrm{~mm}^{2}$ with contraction of $10 \% \pm 5 \%$ at baseline and $19 \% \pm 6 \%$ after calcium $(P=.007)$. Segmental contractility of anterior, posterior, and septal annular regions was $7 \% \pm 5 \%, 6 \% \pm 4 \%$, and $6 \% \pm 3 \%$, respectively. Only anterior region had increased contractility after calcium infusion (to $15 \% \pm 5 \%$; $P=.023)$. Annulus had its high points at anteroseptal commissure and the midposterior region and lowest point in the midseptal region with maximal and minimal height of $5.0 \pm 1.1 \mathrm{~mm}$ and $4.0 \pm 1.1 \mathrm{~mm}$, respectively. The greatest curvature responsible for out of plane annular bending was observed at annular high points.

Conclusions: The human tricuspid annulus is a complex 3-dimensional dynamic structure with its high points and maximal degree of bending at the anteroseptal commissure and midposterior annulus. These detailed geometric data may aid the design of more physiologic annular prostheses and surgical reparative techniques. (J Thorac Cardiovasc Surg 2019;157:1452-61)

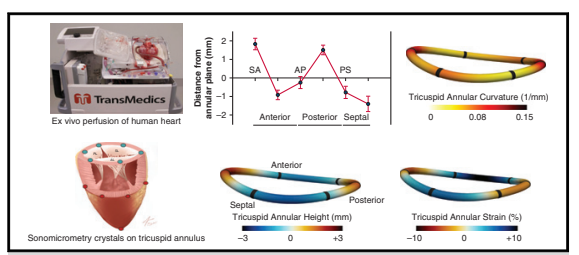

Tricuspid annular height, curvature, and strain calculated based on sonomicrometry crystals.

\section{Central Message}

The human tricuspid annulus is a complex nonplanar structure with distinct high and low points. The geometry of the annulus is stable during the cardiac cycle and not influenced by inotropic stimulation.

\section{Perspective}

Currently used tricuspid annuloplasty prostheses greatly vary in their geometric shape yet lack clear scientific basis to support their design. Detailed geometrical analysis of the human tricuspid annulus along with stress/ strain mapping may therefore drive a more rational design of the shape and material properties of more physiologic annular prostheses.

See Commentaries pages on 1462 and 1463.

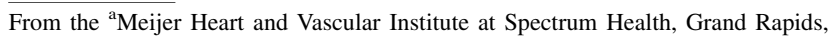
Mich; ${ }^{b}$ Department of Cardiac Surgery, Medical University of Silesia School of

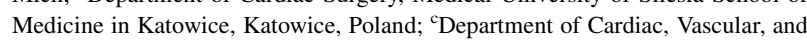
Endovascular Surgery and Transplantology, Medical University of Silesia School of Medicine in Katowice, Silesian Centre for Heart Diseases, Zabrze, Poland; and ${ }^{\mathrm{d}}$ Departments of Aerospace Engineering and Engineering Mechanics and Biomedical Engineering, Institute for Computational Engineering and Science, University of Texas at Austin, Austin, Tex.

Supported by an internal grant from Meijer Heart and Vascular Institute at Spectrum Health. Dr Jovinge received the scientific grant from Richard and Helen DeVos Foundation. Drs Malinowski and Jazwiec are the Peter C. and Pat Cook Endowed Research Fellows in Cardiothoracic Surgery.

Received for publication May 4, 2018; revisions received Aug 23, 2018; accepted for publication Aug 23, 2018; available ahead of print Nov 2, 2018.

Address for reprints: Tomasz A. Timek, MD, PhD, Meijer Heart and Vascular Institute at Spectrum Health, 100 Michigan Ave, SE, Grand Rapids, MI 49503 (E-mail: Tomasz.Timek@spectrumhealth.org).

$0022-5223 / \$ 36.00$

Copyright (c) 2018 by The American Association for Thoracic Surgery

https://doi.org/10.1016/j.jtcvs.2018.08.110
Tricuspid valve (TV) annuloplasty with a prosthetic ring represents the gold standard in contemporary surgical treatment of functional tricuspid regurgitation (FTR). The long-term results of surgical repair of FTR are not satisfactory, with many patients experiencing recurrent regurgitation. ${ }^{1}$ These imperfect results may be partially attributed to our incomplete understanding of the complex geometry and dynamics of the human TV. Filling this

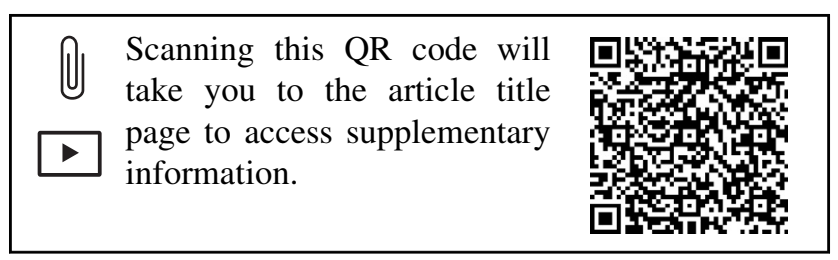




$$
\begin{aligned}
& \text { Abbreviations and Acronyms } \\
& \begin{aligned}
\text { 3D } & =3 \text {-dimensional } \\
\text { AHCWR }= & \text { annular height to commissural width } \\
& \text { ratio } \\
\text { A-P }= & \text { anteroposterior } \\
\text { ED } & =\text { end-diastole } \\
\text { ES } & =\text { end-systole } \\
\text { FTR } & =\text { functional tricuspid regurgitation } \\
\text { OCS } & =\text { Organ Care System } \\
\text { RV } & =\text { right ventricle } \\
\text { S-L } & =\text { septolateral } \\
\text { TA } & =\text { tricuspid annulus } \\
\text { TV } & =\text { tricuspid valve }
\end{aligned}
\end{aligned}
$$

knowledge gap is fundamental to the advancement of current surgical therapy of FTR and to the reduction of recurrent disease. ${ }^{2}$

Several clinical studies have described human tricuspid annulus (TA) geometry utilizing magnetic resonance imaging, ${ }^{3}$ computed tomography, ${ }^{4,5}$ and 2 -dimensional ${ }^{6}$ or 3-dimensional (3D) echocardiography. ${ }^{7-9}$ However, these advanced clinical imaging modalities cannot reliably assess small changes in valvular 3D geometry nor follow discreet annular or subvalvular points in time. Due to morphology of the right ventricle (RV) and complexity of the TV, clinical imaging does not allow for the detailed analysis of individual segments of the TA or the subvalvular apparatus. ${ }^{10} 3 \mathrm{D}$ echocardiography is limited by low frame rate acquisition and inability to record the entire undisturbed heart cycle, ${ }^{9}$ whereas use of magnetic resonance imaging in the 3D assessment of valvular structures is possible only after multiplane reconstructions. ${ }^{3}$ Experimental methodology such as crystal sonomicrometry is much better suited to capture the subtle geometric alterations that are associated with cardiac chamber and valvular remodeling,${ }^{11}$ and this technology has been used to mark specific sites on the TV complex and elucidate detailed valvular geometry and dynamics in animal models. ${ }^{12-14}$ However, sonomicrometry requires surgical implantation of miniature crystals and a wired connection, and therefore cannot be used to study human hearts in normal clinical settings. The aim of the current study was to describe the detailed 3D geometry and dynamics of the human TA utilizing sonomicrometry crystal technology in isolated, working human hearts rejected for clinical transplantation.

\section{METHODS}

The study was evaluated by our local Spectrum Health Internal Review Board and determined as nonhuman research as defined by Department of Health and Human Services and Food and Drug Administration regulations (Spectrum Health Institutional Review Board No. 2016-248).

A total of 11 donor hearts rejected for clinical transplantation with normal RV function and without tricuspid regurgitation were studied.
Hearts were harvested from donors with a mean age of $50 \pm 13$ years and a mean weight of $78 \pm 19 \mathrm{~kg}$. Six donors were men, and the mean left ventricular ejection fraction was $57 \% \pm 4 \%$. None of hearts had more than mild tricuspid regurgitation or right ventricular dysfunction. The average heart cold ischemia time was $118 \pm 67$ minutes. The mean coronary sinus lactate level at the time of data acquisition was $3.7 \pm 1.1 \mathrm{mmol} / \mathrm{L}$ with arterial level at $3.9 \pm 1.0 \mathrm{mmol} / \mathrm{L}$ indicative of cardiac lactic acid absorption and adequate physiologic resuscitation. ${ }^{15}$ No increase in tricuspid regurgitation from preoperative state was observed in the right heart working mode.

\section{Surgical Preparation}

All hearts were harvested using the normal clinical procurement protocol. The hearts were rejected for clinical transplantation due to infection concerns (hepatitis B and C), mild to moderate left system disease on coronary angiography, or mild left ventricle dysfunction. At least $1200 \mathrm{~mL}$ heparinized donor blood was collected in a blood storage system immediately before aortic crossclamping. Subsequently, the aorta was crossclamped and $2000 \mathrm{~mL}$ cold HTK-Custodiol solution (Essential Pharmaceuticals, Durham, $\mathrm{NC}$ ) was delivered in the aortic root for myocardial preservation. After excision, the heart was transported on ice to the experimental site, and subsequently the right atrium was opened and 6 sonomicrometry crystals (Sonometrics Inc, London, Ontario, Canada) were sewn around the TA with 5-0 polypropylene suture (Figure 1); 4 along the mid-RV free wall and 1 at the apex. A solid-state pressure transducer (PA4.5-X6; Konigsberg Instruments Inc, Pasadena, Calif) was placed through the RV apex for RV pressure measurements and 1 in the right atrium for right atrial pressure measurements. The right atriotomy was closed and both superior and inferior vena cava oversewn. The aorta was sized and cannulated with a connector and the pulmonary artery was instrumented with a $30 \mathrm{~F}$ cannula in preparation for the connection to the Organ Care System (OCS) (TransMedics, Andover, Mass).

The OCS is a portable ex vivo organ perfusion platform used in clinical transplantation and designed to perfuse the beating, unloaded donor heart with warm, oxygenated blood during transport to the transplant center (Figure 1). The OCS device was primed with donor blood in accordance to the clinical protocol and the heart mounted on the device, defibrillated if spontaneous rhythm did not return, ventricularly paced at $80 \mathrm{bpm}$, and perfused under normothermic conditions. The heart was perfused with a mean arterial blood pressure of $75 \mathrm{~mm} \mathrm{Hg}$ in the clinical unloaded mode. The pumped blood to the aorta constituted the inflow of the oxygenated blood to the heart. Through the coronary arteries it reached the coronary sinus and served as the inflow to the right heart. The blood was subsequently ejected by the RV to the pulmonary artery outflow cannula and collected in the venous reservoir. Arterial (aortic inflow) and coronary sinus venous (pulmonary artery cannula) blood gases, including lactate levels, were collected every 15 minutes to assure stability of the experimental preparation. ${ }^{15}$

\section{Data Acquisition}

After 60 minutes of reperfusion, the outflow pulmonary artery cannula was partially occluded allowing the right heart to be filled with blood. The pulmonary artery was narrowed to achieve maximal RV pressure of $30 \mathrm{~mm}$ $\mathrm{Hg}$, thus converting to the right heart working mode. TV competence was assessed with epicardial echocardiography utilizing a Vivid S6 machine (GE Healthcare, Chicago, Ill).

Hemodynamic and sonomicrometry data were acquired simultaneously under working right heart conditions (baseline $\mathrm{n}=11$ ). The last 5 hearts in the study were additionally tested before (precalcium) and after (calcium) $500 \mathrm{mg}$ calcium chloride bolus infusion to assess the changes in annular geometry and strain under contractile stimulation.

\section{Sonomicrometric Data}

All sonomicrometry data were acquired using a Sonometrics Digital Ultrasonic Measurement System DS3 (Sonometrics Inc) as 

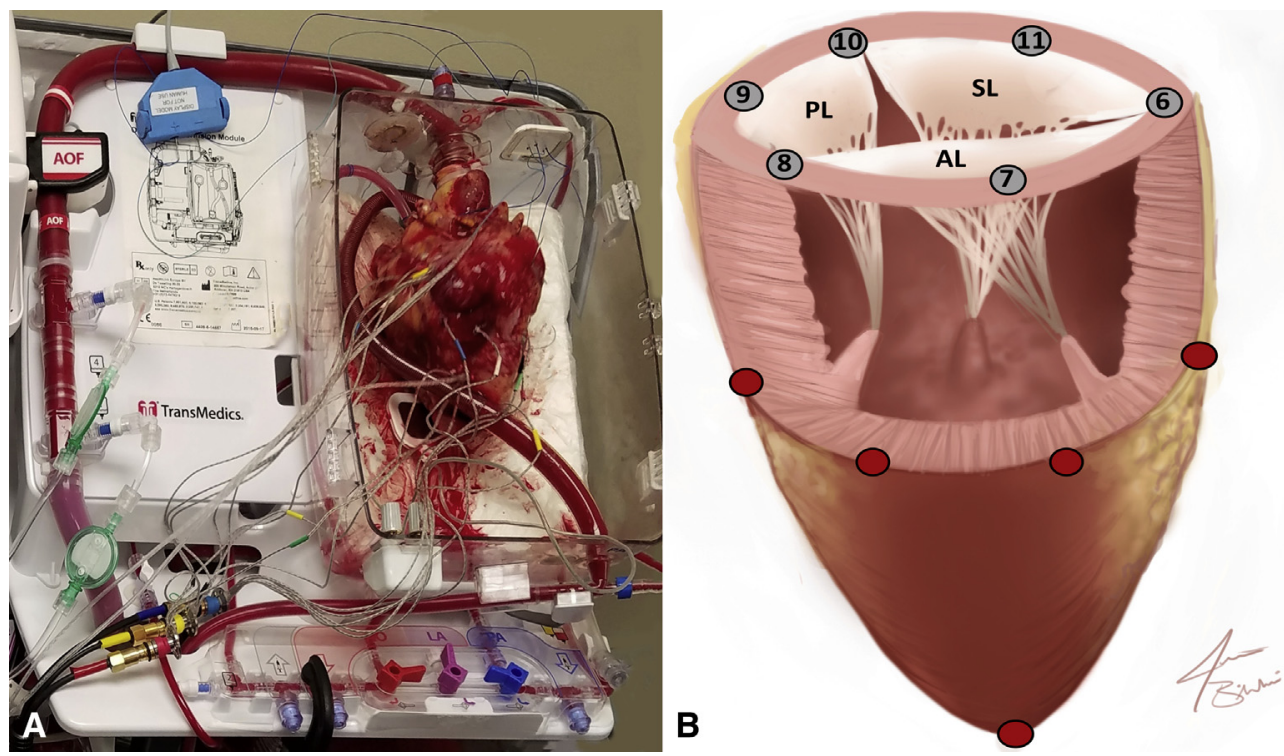

FIGURE 1. A, Experimental setup demonstrating 1 of the study hearts mounted on the ex vivo perfusion unit of the Organ Care System (TransMedics, Andover, Mass). B, Location of sonomicrometry crystals implanted around the tricuspid annulus and on the right ventricle. $P L$, Posterior leaflet; $A L$, anterior leaflet; $S L$, septal leaflet.

previously described. ${ }^{14}$ Data from 3 consecutive cardiac cycles during hemodynamically stable conditions (Table E1) were averaged for each heart. Given that all 3 cardiac cycles were recorded in immediate succession, there were no physiologic or external reasons for changes in the signal. Therefore, averaging consecutive cardiac cycles increased the signal to noise ratio and made data more reliable. Data were acquired at $128 \mathrm{~Hz}$ with simultaneous RV pressure and right atrial pressure, and electrocardiogram recordings. The time-resolved 3D coordinates of the crystals were obtained from the SonoSoft software (Sonometrics Inc) and analyzed in Matlab (MathWorks, Natic, Mass) using customdesigned software. Least-squares cubic splines were fit to the discrete sonomicrometry annular crystal data to create approximations of the TA at each time point and for each heart. ${ }^{16}$ Spline-based annular metrics add to the standard distance-based sonomicrometry analysis by allowing to spatially resolve the configurational changes of the annulus as it undergoes complex in-plane and out-of-plane deformations during the cardiac cycle. This spatially continuous mathematical description was used to obtain complete height profile of the TA. Moreover, the annular 3D geometry was also expressed as the maximal displacement of annular crystals from annular best-fit plane. Annular height to commissural width ratio (AHCWR) was calculated as the ratio of maximal displacement of annular crystals from annular plane (height) to its anteroposterior (A-P) diameter. Annular area and perimeter were calculated based on the least-squares cubic splines. The septolateral (S-L) annular dimension was calculated as the distance between crystal 11 and the midpoint between crystals 7 and 8; the A-P annular dimension was calculated as the distance between crystals 6 and 9. The eccentricity index was calculated as the A-P to S-L distance ratio. Intercommisural distances were calculated as the respective distances between crystals 6, 8, and 10. Annular contraction was computed as the difference between the maximal and minimal TA area $\left(\left[A_{\max }-A_{\min }\right] / A_{\min } \times 100 \%\right)$. Sonomicrometry-derived RV contraction (ie, fractional area change) was calculated as the difference between the maximal and minimal area enclosed by crystals 1 to $4\left(\left[\mathrm{~A}_{\mathrm{ED}}-\mathrm{A}_{\mathrm{ES}}\right] /\right.$ $\mathrm{A}_{\mathrm{ED}} \times 100 \%$ ) and served as a surrogate of RV performance. $\mathrm{RV}$ volume was assessed using convex hull method.

All values were calculated at end-diastole (ED), end-systole (ES), end of isovolumic contraction, end of isovolumic relaxation, maximal valve opening (defined as the time when annular area was the largest), minimal valve opening (the time when annular area was the smallest). ED was defined as the time of the beginning of the positive deflection in electrocardiogram voltage ( $\mathrm{R}$ wave), whereas ES was determined to be the time of maximum negative $\mathrm{dP} / \mathrm{dt}$ of the $\mathrm{RV}$ pressure. End of isovolumic contraction and end of isovolumic relaxation were determined as end points of the linear RV pressure segments during isovolumetric contraction and relaxation, respectively.

\section{Annular Strain and Curvature Analysis}

TA strain was calculated based on the first spatial derivative of the spline fits using the method previously described. ${ }^{16,17}$ Strain, as a relative measure of displacement and thus annular deformation, is calculated relative to a reference configuration. Baseline at ED and at maximal valve opening chosen as the reference configurations for other time points within the cardiac cycle. For the last 5 hearts in the study, we also compared strains after calcium stimulation with precalcium configuration used as the reference for the same time points during calcium infusion. Therefore, cardiac strain first reflects the deformation of the annulus in baseline conditions and intervention strain reflects the deformation of the annulus induced after calcium. Specifically, GreenLagrange strain was calculated along the annulus for each heart and later displayed on a spline representation of the population-averaged annulus. The global and regional strain patterns were calculated for the entire annular circumference (crystals 6-11) and the anterior (crystals 6-8), posterior (crystals 8-10), and septal (crystals 10-6) regions separately. Negative or compressive strains imply that tissue is contracted, whereas positive or tensile strains imply that tissue is stretched. The absolute strains have positive values only and are indicative of annular deformation. Local annular curvature was calculated based on the principles previously presented in detail. ${ }^{16}$

\section{Statistical Analysis}

Data are presented as mean \pm standard deviation unless otherwise stated. The baseline data shown are predominantly descriptive. The within heart cycle comparisons and the precalcium and calcium conditions were tested with the use of Student $t$ test for dependent observations or Wilcoxon 

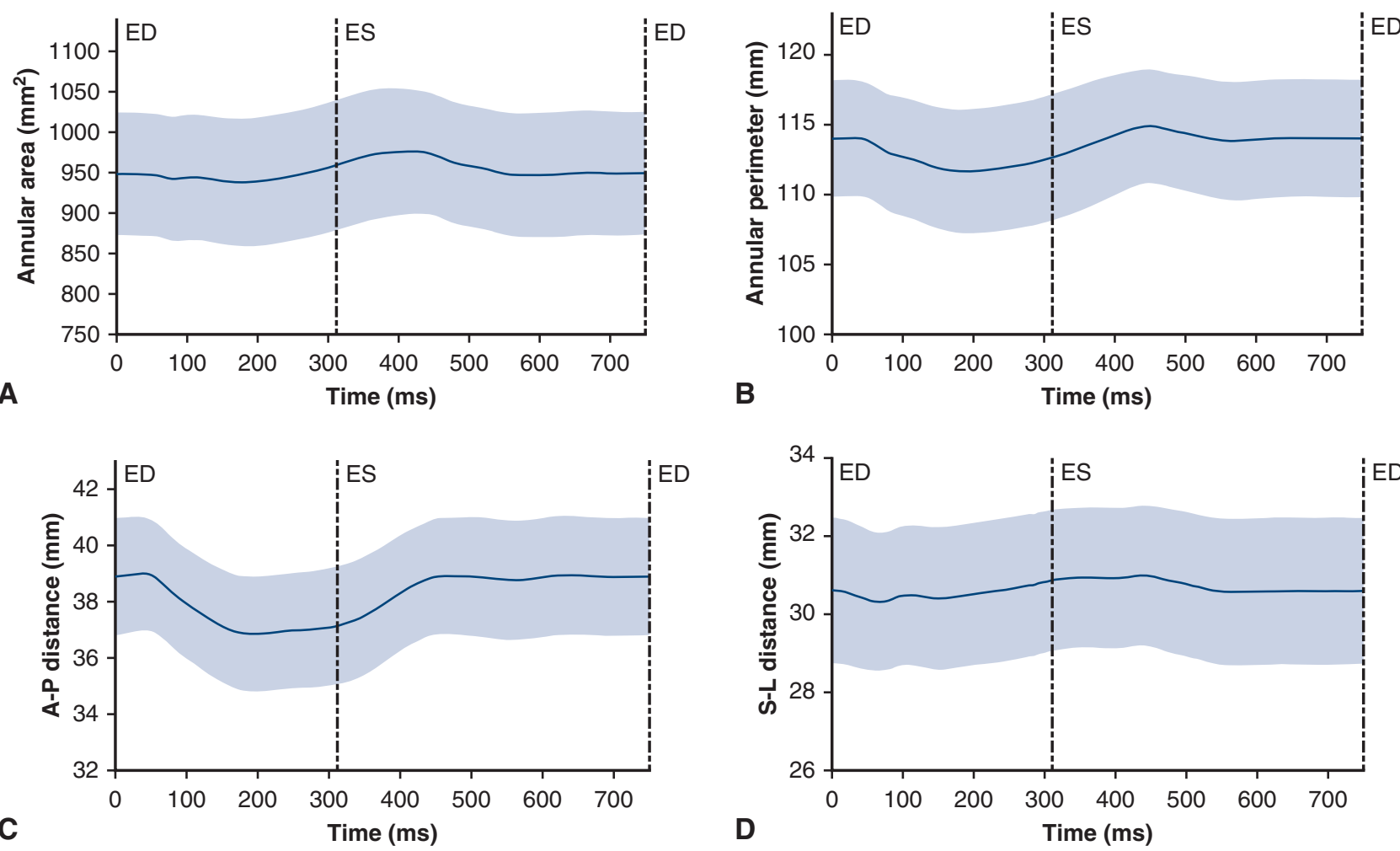

FIGURE 2. Group mean data for tricuspid annular metrics during the averaged cardiac cycle for baseline $(\mathrm{n}=11)$. Data are presented as mean \pm standard error of the mean. A, Annular area. B, Annular perimeter. C, Anteroposterior $(A-P)$ annular distance. D, Septolateral $(S-L)$ annular distance. $E D$, End-diastole; $E S$, end-systole.

signed-rank test for repeated measurements when the normality test failed (SigmaPlot 12.5; Systat, San Jose, Calif).

\section{RESULTS}

\section{Hemodynamic Parameters}

During baseline conditions, mean heart rate was $80 \pm 2$ bpm, maximal RV pressure was $28 \pm 4 \mathrm{~mm} \mathrm{Hg}$, RV enddiastolic pressure was $8 \pm 3 \mathrm{~mm} \mathrm{Hg}$, and right atrial pressure was $6 \pm 3 \mathrm{~mm} \mathrm{Hg}$. Hemodynamic variables before and after calcium infusion are presented in Table E2. The expected increase in the RV fractional area change was noted after stimulation with calcium with no difference in other measured parameters.

\section{TA Size}

The maximal area of the TA in diastole was $997 \pm 258 \mathrm{~mm}^{2}$ and minimal area during systole was $902 \pm 257 \mathrm{~mm}^{2}$. The maximal perimeter was $115 \pm 14 \mathrm{~mm}$ and minimal perimeter was $110 \pm 14 \mathrm{~mm}$. The S-L distance at the maximal valve opening was $30.8 \pm 5.7 \mathrm{~mm}$ and A-P diameter was $39.4 \pm 6.6 \mathrm{~mm}$. At minimal valve opening, S-L diameter was $28.7 \pm 5.9 \mathrm{~mm}$ and A-P diameter was $36.4 \pm 6.9 \mathrm{~mm}$. Annular area and dimensions throughout the cardiac cycle at Baseline are shown in Figure 2. Values for annular distances before and after calcium infusion are illustrated in Table 1. Of note is the disproportionate diastolic enlargement between A-P and S-L diameter. The eccentricity index increased from $0.76 \pm 0.19$ in diastole to $0.85 \pm 0.19$ during systole at baseline $(P=.007)$. There was no change in annular eccentricity after calcium, and within-heart cycle dynamics remained unaffected: $0.78 \pm 0.21$ during diastole and $0.92 \pm 20$ in systole $(P=.029)$.

\section{Annular Geometry}

The 3D structure of the reconstructed annulus revealed its high points at the anteroseptal commissure and the midposterior annulus, whereas low points were observed in the septal and the A-P region as demonstrated in Figure 3. Annular height changed dynamically during the heart cycle by $20 \% \pm 10 \%$ with maximal height of $5.0 \pm 1.1 \mathrm{~mm}$ and minimal height of $4.0 \pm 1.1 \mathrm{~mm}$. Annular height and curvature maps at baseline are presented in Figure 4. Video 1 illustrates the annular height during 3 recorded heart cycles at baseline. Calcium stimulation did not alter annular height, maintaining the maximal height at $5.4 \pm 1.3 \mathrm{~mm}$ and minimal height at $4.0 \pm 1.5 \mathrm{~mm}(P=.9$ and $P=.51 \mathrm{vs}$ precalcium, respectively). AHCWR at baseline was $12 \% \pm 5 \%$ at maximal valve opening and $13 \% \pm 6 \%$ at its minimum. No significant effect on AHCWR was observed with calcium at either maximal and minimal valve opening. 
TABLE 1. Tricuspid annular regional diameters and dynamics

\begin{tabular}{|c|c|c|c|c|c|c|c|c|c|}
\hline \multirow[b]{2}{*}{ Variable } & \multicolumn{3}{|c|}{ Max } & \multicolumn{3}{|c|}{ Min } & \multicolumn{3}{|c|}{ Contraction $(\%)$} \\
\hline & Precalcium & Calcium & $P$ value & Precalcium & Calcium & $P$ value & Precalcium & Calcium & $P$ value \\
\hline Anterior annulus (mm) & $48.9 \pm 3.8$ & $46.8 \pm 4.1$ & .075 & $44.6 \pm 6.4$ & $40.1 \pm 6.3$ & .019 & $9 \pm 6$ & $15 \pm 6$ & .023 \\
\hline $6-7$ & $25.8 \pm 2.6$ & $24.5 \pm 3.3$ & .088 & $23.4 \pm 3.3$ & $21.0 \pm 4.0$ & .009 & $10 \pm 6$ & $15 \pm 7$ & .001 \\
\hline $7-8$ & $23.6 \pm 3.3$ & $23.1 \pm 3.9$ & .360 & $21.0 \pm 4.4$ & $18.7 \pm 5.3$ & .089 & $12 \pm 9$ & $21 \pm 10$ & .050 \\
\hline Posterior annulus (mm) & $23.9 \pm 5.8$ & $22.5 \pm 5.6$ & .066 & $22.2 \pm 6.4$ & $20.1 \pm 5.5$ & .017 & $8 \pm 4$ & $11 \pm 3$ & .114 \\
\hline $8-9$ & $11.4 \pm 3.5$ & $10.7 \pm 3.3$ & .048 & $10.1 \pm 4.0$ & $8.9 \pm 3.5$ & .017 & $10 \pm 8$ & $20 \pm 9$ & $<.001$ \\
\hline $9-10$ & $12.7 \pm 2.5$ & $12.2 \pm 2.2$ & .078 & $11.9 \pm 2.8$ & $10.8 \pm 2.3$ & .013 & $7 \pm 4$ & $12 \pm 3$ & $<.001$ \\
\hline Septal annulus (mm) & $33.9 \pm 5.3$ & $33.1 \pm 4.8$ & .300 & $31.9 \pm 4.2$ & $30.4 \pm 3.4$ & .091 & $6 \pm 3$ & $8 \pm 5$ & .110 \\
\hline $10-11$ & $17.4 \pm 1.6$ & $17.2 \pm 1.3$ & .132 & $16.7 \pm 1.3$ & $16.1 \pm 0.9$ & .177 & $4 \pm 3$ & $6 \pm 4$ & .232 \\
\hline $11-6$ & $16.4 \pm 4.2$ & $15.9 \pm 4.0$ & .392 & $15.2 \pm 3.5$ & $14.3 \pm 3.1$ & .048 & $7 \pm 4$ & $9 \pm 7$ & .398 \\
\hline \multicolumn{10}{|l|}{$\mathrm{C}-\mathrm{C}(\mathrm{mm})$} \\
\hline $6-8$ & $36.4 \pm 4.2$ & $35.9 \pm 3.9$ & .142 & $32.1 \pm 3.5$ & $29.7 \pm 3.2$ & .056 & $11 \pm 10$ & $17 \pm 10$ & .049 \\
\hline $6-10$ & $27.3 \pm 4.5$ & $26.8 \pm 4.1$ & .062 & $25.7 \pm 3.5$ & $24.4 \pm 3.6$ & .124 & $6 \pm 5$ & $9 \pm 5$ & .246 \\
\hline $8-10$ & $20.8 \pm 3.3$ & $19.2 \pm 2.8$ & .084 & $19.2 \pm 3.5$ & $17.1 \pm 2.7$ & .028 & $8 \pm 4$ & $11 \pm 4$ & .096 \\
\hline S-L diameter (mm) & $28.6 \pm 4.3$ & $26.9 \pm 3.6$ & .114 & $25.6 \pm 4.8$ & $22.7 \pm 4.3$ & .009 & $11 \pm 7$ & $16 \pm 8$ & .011 \\
\hline A-P diameter (mm) & $34.3 \pm 5.7$ & $34.0 \pm 5.7$ & .269 & $31.0 \pm 5.0$ & $29.0 \pm 4.0$ & .052 & $9 \pm 4$ & $14 \pm 7$ & .079 \\
\hline
\end{tabular}

Data calculated based on 3-dimensional crystal coordinates and presented as mean \pm standard deviation $(\mathrm{n}=5)$. Anterior, posterior, and septal regional distances and annular dimensions calculated based on spline curves. Crystal numbers as depicted in Figure 1. Max, Maximal distance in diastole; Min, minimal distance in systole; $C$ - $C$, intercommisural distance; $S-L$, septolateral; $A-P$, anteroposterior.

\section{TA Dynamics}

The TA dynamically changed its dimensions during the heart cycle with an area change of $10 \% \pm 5 \%$ and perimeter change of $5 \% \pm 2 \%$ at baseline, and $19 \% \pm 6 \%$ and

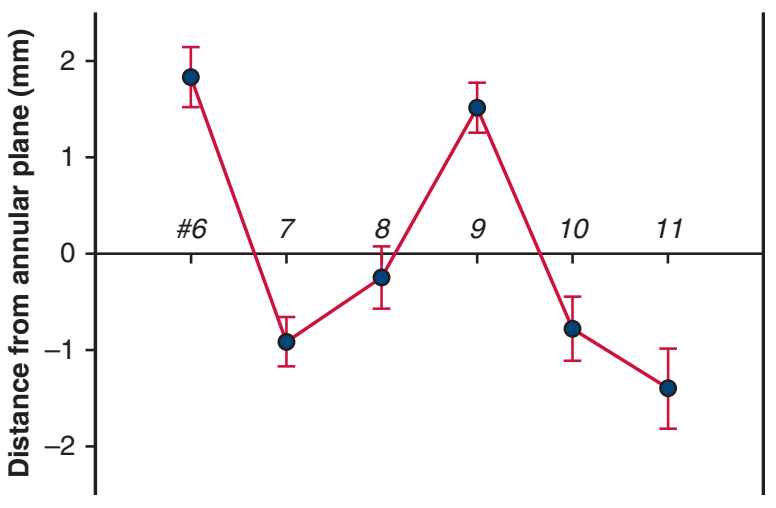

A

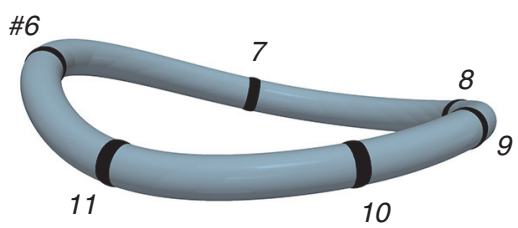

B

FIGURE 3. Tricuspid annular geometry at maximal valve opening during baseline $(\mathrm{n}=11)$. A, Orthogonal distance of each annular crystal to the best-fit annular plane. Data are presented as mean \pm standard error of the mean. B, Average spline fit representation of the tricuspid annulus. Crystal numbers as presented in Figure 1 with crystal 6 representing anteroseptal, crystal 8 representing anteroposterior, and crystal 10 representing posteroseptal commissure.
$8 \% \pm 3 \%$ after calcium, respectively. The annulus presented uniform dynamics across all its segments at baseline. From diastole to systole, the anterior annulus contracted by $7 \% \pm 5 \%$ from $49.5 \pm 5.6 \mathrm{~mm}$ to 46.1 $\pm 6.7 \mathrm{~mm}(P<.001)$, the posterior annulus by $6 \% \pm 4 \%$ from $28.3 \pm 6.9 \mathrm{~mm}$ to $26.6 \pm 7.1 \mathrm{~mm}(P<.001)$, and the septal annulus by $6 \% \pm 3 \%$ from $39.4 \pm 8.5 \mathrm{~mm}$ to $36.8 \pm 7.5 \mathrm{~mm}(P<.001)$. Regional annular segment lengths and intercommissural distances before and after calcium stimulation are presented in Table 1.

\section{Annular Strains}

The TA showed significant deformation during the heart cycle with mean annular strain of $-4 \% \pm 2 \%$ at TV closure $(P<.001$ vs opening $)$. All annular segments presented with compression during valve closure with largest at anterior annulus $(-5 \% \pm 4 \%)$ and posterior annulus $(-6 \% \pm 3 \%)$ and smallest at the septal annulus $(-2 \% \pm 2 \%)$ (all $P$ values $<.05$ vs valve opening). Annular strain at baseline during 3 consecutive heart cycles is presented in Video 2. Absolute annular strains showing annular deformation in relation to ED across all regions are shown in Figure 5. The strain pattern with calcium infusion revealed heterogonous strain along the annulus with the minimal compression in the septal portion and the largest contraction in the anterior annulus close to the anteroseptal commissure (Figure 6).

\section{DISCUSSION}

The TA is the main therapeutic target in the surgical treatment of FTR, and its detailed morphologic mapping 

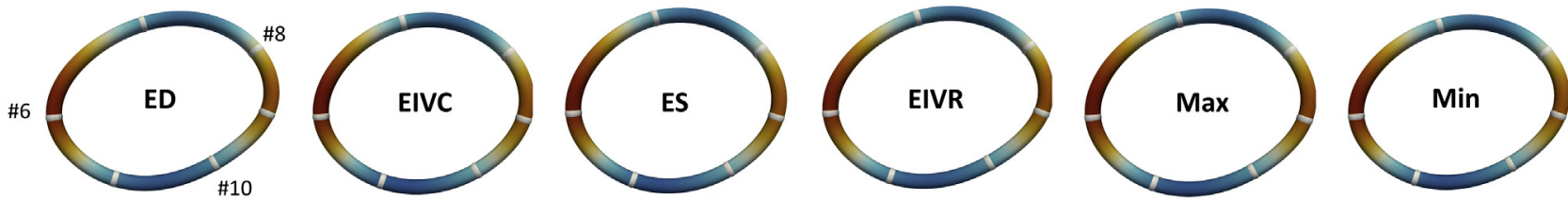

Height $(\mathrm{mm})$

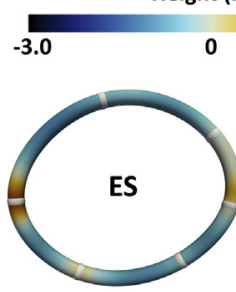

0

$+3.0$
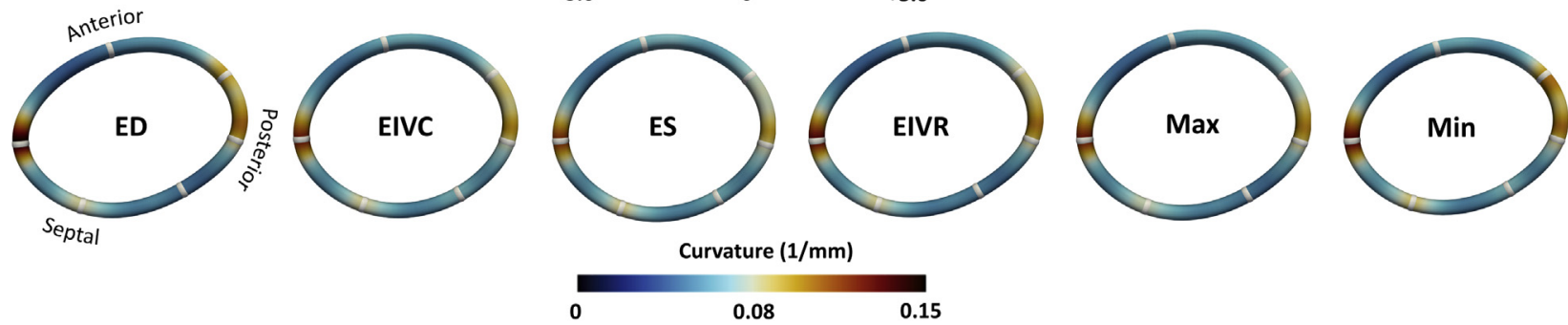

FIGURE 4. Top panel, Tricuspid annular height map at baseline $(\mathrm{n}=11)$. Bottom panel, Curvature map at baseline $(\mathrm{n}=11)$. The metrics are shown on the average annular spline. Height is presented as the distance from the best-fit tricuspid annular plane. Crystals 6 , 8 , and 10 represent anteroseptal, anteroposterior, and posteroseptal commissures, respectively. ED, End-diastole; EIVC, end of isovolumic contraction; ES, end-systole; EIVR, end of isovolumic relaxation; Max, maximal valve opening; Min, minimal valve opening.

is critical to the design of annuloplasty prostheses that may better re-establish the physiologic geometry of the diseased valve. In the current study, we describe for the first time the detailed 3D geometry of the human TA using sonomicrometry crystals to mark discreet annular sites. We found the TA to be a complex 3D structure with its high points near the anteroseptal commissure and the midposterior annulus and low points in the midanterior and midseptal segments. The area of highest curvature was observed at the anteroseptal commissure and midposterior annulus. This complex spatial configuration was not altered by contractile stimulation with calcium.

Initial human cadaver studies of the TA described it as a flat structure. ${ }^{18}$ In 2004, Hiro and colleagues ${ }^{12}$ implanted sonomicrometry crystals onto the TV complex in sheep

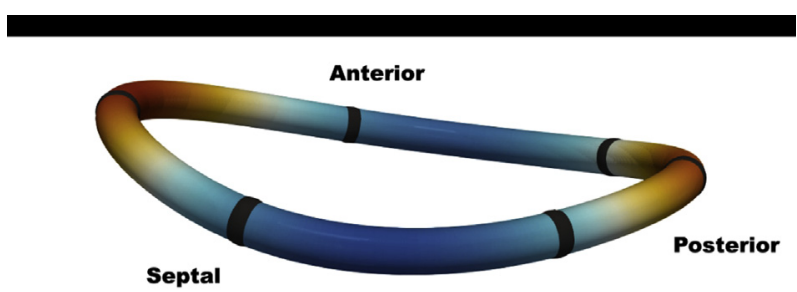

Tricuspid Annular Height (mm)

$\begin{array}{lll}-3 & +3\end{array}$

VIDEO 1. Tricuspid annular height at baseline. Height is expressed as the maximal displacement from the annular best-fit plane. Video available at: https://www.jtcvs.org/article/S0022-5223(18)32505-4/fulltext. and showed the annulus to be a saddle-shaped structure with local maxima at the posterior annulus, the anteroseptal commissure, and the anterior annulus and minima at A-P and posteroseptal commissures. Together with its intricate geometry, the annulus was described to have an elliptical shape, becoming more circular during maximal valve opening. These results were later corroborated using the same experimental technology in pigs, ${ }^{13}$ and are consistent with findings reported by our laboratory ${ }^{11,12,17}$ and other investigators. ${ }^{18}$ The current human data support these experimental findings and suggest preservation of the TA shape across mammalian physiology.

Using clinical imaging, the human TA has also been described as nonplanar structure with an oval shape ${ }^{8}$ with multiple studies reporting the geometry of the TA using 3D echocardiography, ${ }^{7,9,19}$ magnetic resonance

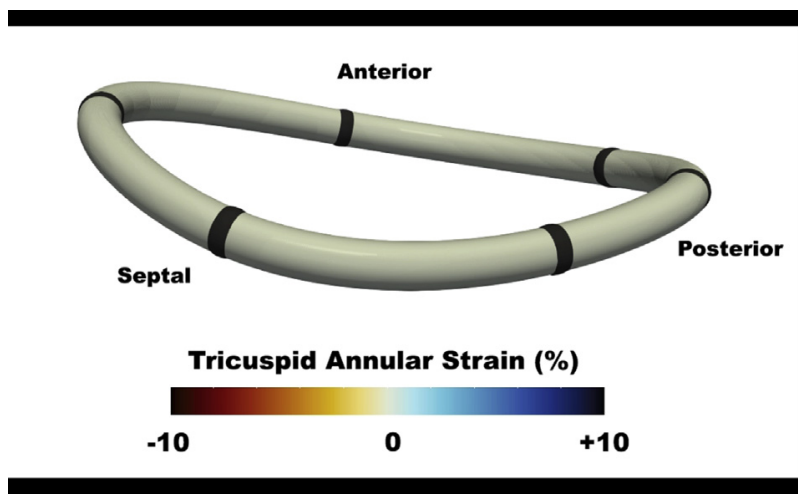

VIDEO 2. Tricuspid annular strain at baseline. Video available at: https:// www.jtcvs.org/article/S0022-5223(18)32505-4/fulltext. 


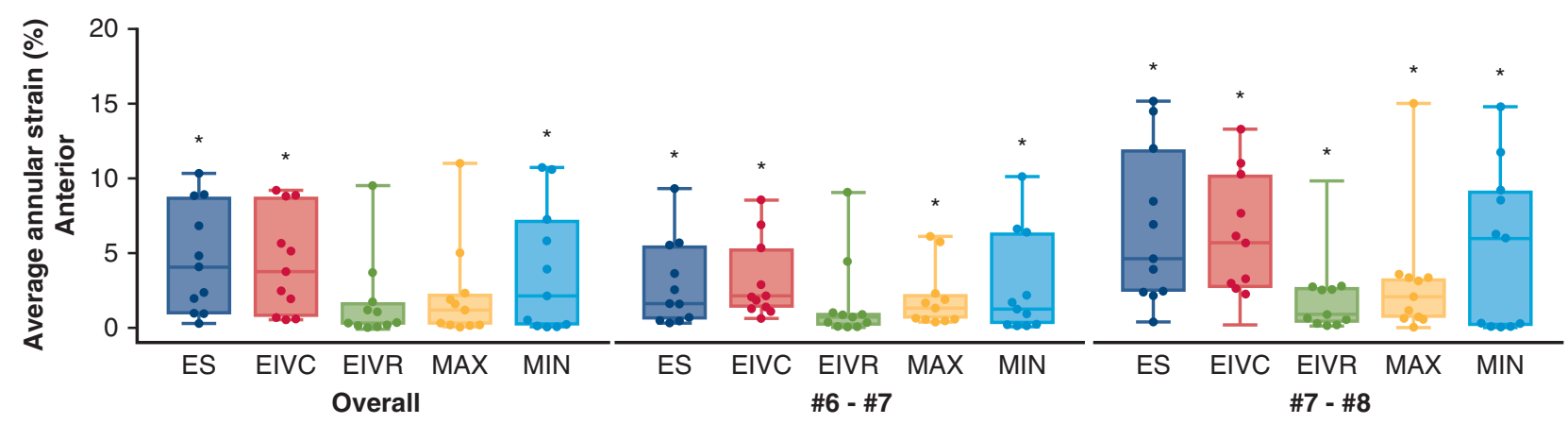

A
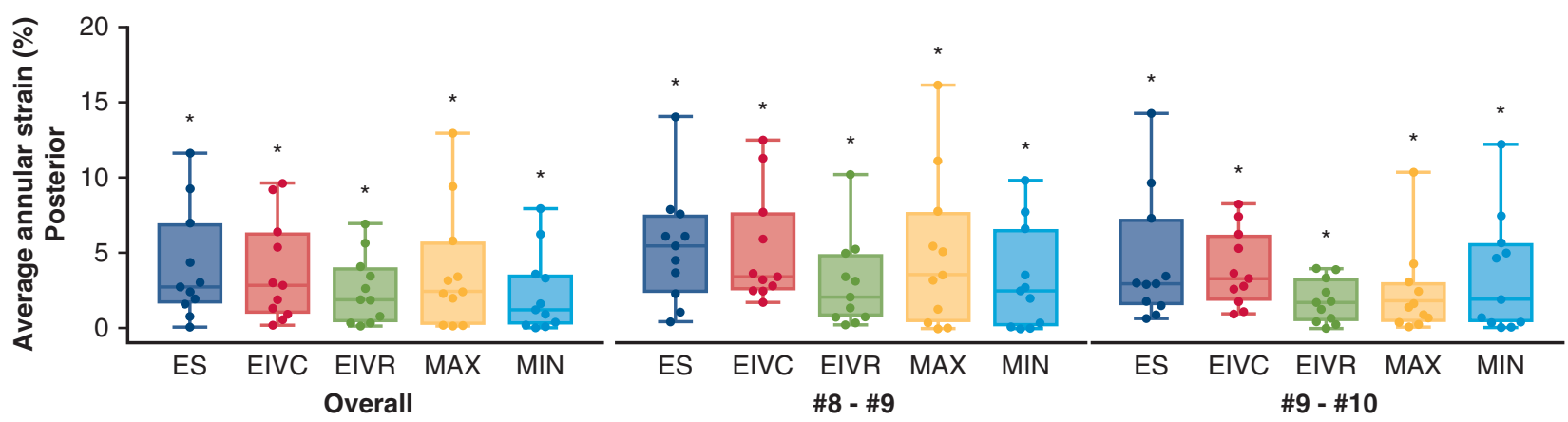

B

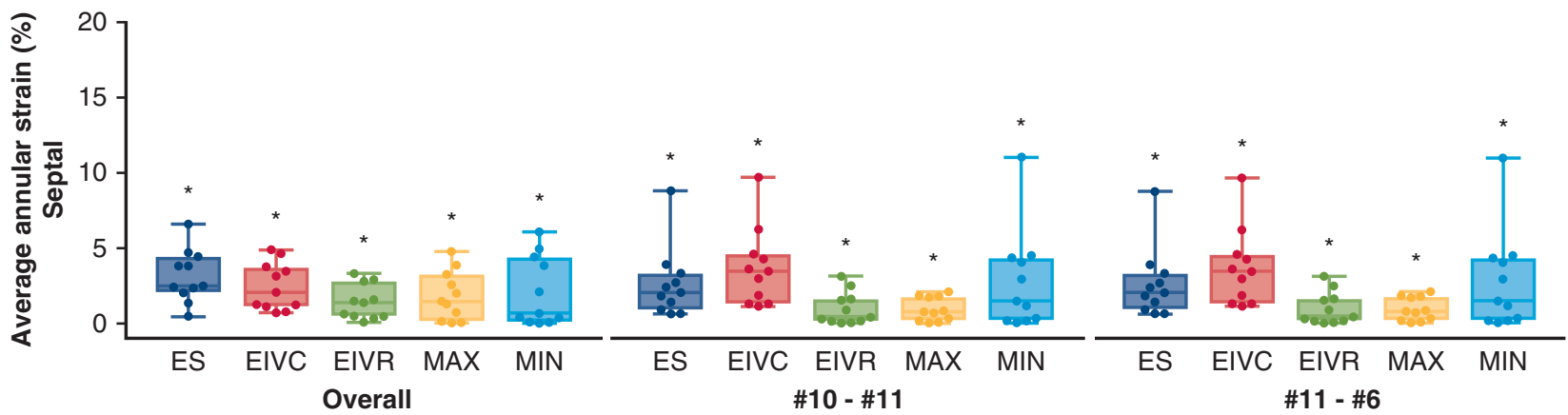

C

FIGURE 5. Regional absolute average annular cardiac strain. The strain is calculated relative to end-diastole at baseline $(\mathrm{n}=11)$. The absolute strains are positive in value and do not reflect stretching or shortening. Crystal numbers defining annular segments as presented in Figure 1. A, Anterior annulus. B, Posterior annulus. C, Septal annulus. The upper and lower borders of the box represent the upper and lower quartile. The middle horizontal line represents the median. The upper and lower whiskers represent the maximum and minimum values. ES, End-systole; EIVC, end of isovolumic contraction; $E I V R$, end of isovolumic relaxation; $M A X$, time of maximal annular area; $M I N$, time of minimal annular area. $* P<.05$ versus end-diastole.

imaging, ${ }^{3,20}$ and computed tomography. ${ }^{4}$ The details of the saddle-like shape of the annulus vary between reports. There appears to be a consensus that, similar to current findings, the highest annular point is at the anteroseptal commissure, ${ }^{7,19}$ but some reports present the highest point at the posterior annulus only.

The saddle geometry of the human TA mirrors the well investigated shape of the mitral annulus, ${ }^{21}$ which was demonstrated to be potentially beneficial in reducing mitral leaflet stress/strain. ${ }^{21,22}$ Yet this may not be the case with the TA because in vitro studies revealed no difference in tricuspid leaflet stretch with either a flat or a saddleshaped annulus. ${ }^{23}$ AHCWR calculated in the current study for the human TA (around 10\%-15\%) was at a level where a $5 \%$ to $13 \%$ peak leaflet strain reduction could be expected if one were to extrapolate available mitral data. ${ }^{21,22}$ However, the TV is more complex and only direct in vivo leaflet strains measurements can provide a definite answer to this important question. The TA undergoes dynamic changes during the heart cycle with maximal area and perimeter in diastole and minimal area and perimeter in midsystole, ${ }^{9,13}$ and we observed a similar pattern for 

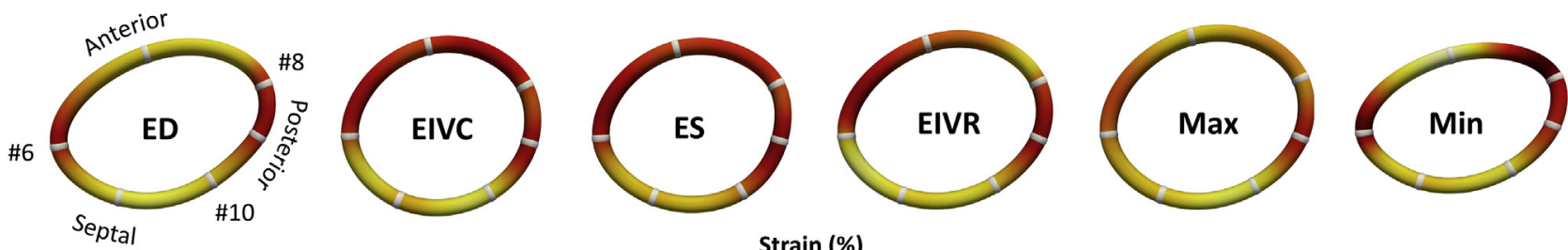

Strain (\%)

$-15$

0

2

FIGURE 6. Tricuspid annular strain maps after calcium administration $(n=5)$. The strain is calculated relative to the pre-calcium conditions at each time point. $E D$, End-diastole; $E I V C$, end of isovolumic contraction; $E S$, end-systole; EIVR, end of isovolumic relaxation; MAX, time of maximal annular area; $M I N$, time of minimal annular area.

annular area and perimeter change in our study. However, the magnitude of annular area reduction during the cardiac cycle of approximately $10 \%$ was lower than previously reported. Tsakiris and colleagues ${ }^{24}$ demonstrated canine tricuspid annular reduction of $39 \%$ during the cardiac cycle, whereas sonomicrometry-derived annular area contraction of $21.9 \%{ }^{12}$ and $21 \%^{13}$ has been reported in sheep and pigs, respectively. Clinically, tricuspid annular area was shown to decrease $29.6 \%$ to $35 \%$ during the heart cycle as measured by transthoracic echocardiography. The lower annular contraction observed in our study may be attributed to the experimental preparation of isolated, denervated hearts subjected to cold ischemic storage and without normal physiologic flow. However, the hearts used in our study remained responsive to contractile stimulation with calcium, and biochemical analysis suggested lactate absorption indicative of adequate myocardial resuscitation. ${ }^{15}$ Our results are comparable to the 3D transesophageal echocardiography study of Owais and colleagues ${ }^{19}$ in anesthetized patients undergoing coronary artery bypass surgery who reported tricuspid annular area contraction of $10 \%$. Furthermore, the degree of TA contraction seen in the current study is similar to clinical ${ }^{25}$ and experimental ${ }^{26}$ area reduction of the mitral annulus.

The most responsive regions of the TA in isolated human hearts were anterior and midposterior segments. These portions of the annulus coincided with locations of the highest annular curvature, presenting as the sites of out-of-plane annular folding. In-plane diameter analysis revealed that changes in the A-P dimension were the primary drivers of the annular ellipse becoming more circular during the cardiac cycle, as reported previously by others. ${ }^{9}$ Limited S-L dynamics may be explained by the characteristics of the septal annulus and the more distensible RV free wall encircling most of the posterior annulus. The conformation changes in both in- (planar dimensions) and out-of- (height, curvature) plane human TA geometry were very similar to the mechanics of the ovine annulus reported by our group previously. ${ }^{16}$
The least responsive portion of the TA to inotropic stimulation was the septal region, with strain of only $-2 \%$ after calcium infusion. These findings may be explained by high stiffness of this segment due to its dense collagen content, ${ }^{27}$ although the septal annulus has previously been shown to be equally susceptible to annular dilation as other regions. ${ }^{7,14}$ Because the left ventricle was unloaded in our experimental preparation, it is feasible that septal contribution to annular dynamics was reduced by diminished interventricular interaction. However, we have previously shown in the ovine model that unloading the left ventricle in vivo has minimal effect of annular geometry or dynamics. ${ }^{28}$

\section{Clinical Significance}

The results of our study may have important clinical implications. Currently used tricuspid annuloplasty prostheses greatly vary in their geometric shape yet lack clear scientific basis to support their design. Most commonly implanted 3D annuloplasty rings incorporate axial heights from $9^{29}$ to $15 \mathrm{~mm},{ }^{30}$ which is significantly greater than the approximate 5- to 6-mm annular height of the normal human TAs reported herein. Detailed geometrical analysis of a human TA along with stress/strain mapping may therefore drive a more rational design of the shape and material properties of more physiologic annular prostheses. Specifically, strain and curvature data may aid in a detailed, spatially resolved understanding of annular dynamics. Strain and curvature changes throughout the cardiac cycle could thus inspire devices with differential stiffness to accommodate the native motion of the valve. This is of utmost importance for improved clinical results and solidifying the concept of annular remodeling in the treatment of functional tricuspid regurgitation. ${ }^{31}$

\section{Limitations of the Study}

The results of our study must be interpreted in the context of important limitations. This study was performed in a small number of isolated human hearts, and the RV working mode was induced by increased RV afterload. Moreover, the use of ex vivo retrograde perfusion system keeps the left 
ventricle unloaded throughout the entire experiment and excludes the influence of interventricular dependence. However, we have demonstrated previously that unloading the left ventricle in vivo does not alter the geometry of the TAs. ${ }^{28}$ Denervation of the heart and need for ventricular pacing may have influenced the dynamic behavior of the TA but would be unlikely to affect annular geometry, which was the main focus of this study. Similarly, lack of cardiac output at the physiologic level through the RV may be expected to influence annular contraction but have a limited effect on its 3D shape. Because of protocol change during the course of the study, only the 5 last hearts were tested before and after calcium stimulation. We chose to analyze 3 consecutive heart cycles to provide more reliable averaged data for all presented parameters. Although this is a short data acquisition time, we believe the results are reliable due to the stable condition of the experimental setup (Table E1). The same number of heart cycles was previously shown to be sufficient in a similar mitral valve study. ${ }^{17}$ The current study analyzed only the TA and did not investigate other key components of the valvular complex such as the valve leaflets and the subvalvular apparatus.

\section{CONCLUSIONS}

Using crystal sonomicrometry in isolated beating hearts, we found the human TA to be a complex nonplanar structure with distinct high points around the anteroseptal commissure and midposterior annulus with low points in the septal and the A-P regions. The TA maintained a saddle-like shape with out-of-plane bending and highest curvature at the anteroseptal commissure. The $3 \mathrm{D}$ geometry of the annulus was stable during the cardiac cycle and not influenced by inotropic stimulation. The planar shape of the annulus at ED resembled an ellipse that became more circular during ES. These geometric findings (shown in the Graphical Abstract) should be considered in the design of more physiologic TA prostheses.

\section{Conflict of Interest Statement}

Authors have nothing to disclose with regard to commercial support.

TransMedics Inc (Andover, Mass) provided the Organ Care System for research use and technical support during the experiments.

\section{References}

1. McCarthy PM, Bhudia SK, Rajeswaran J, Hoercher KJ, Lytle BW, Cosgrove DM, et al. Tricuspid valve repair: durability and risk factors for failure. J Thorac Cardiovasc Surg. 2004;127:674-85.

2. Fukuda S, Gillinov AM, McCarthy PM, Matsumura Y, Thomas JD, Shiota T. Echocardiographic follow-up of tricuspid annuloplasty with a new three-dimensional ring in patients with functional tricuspid regurgitation. J Am Soc Echocardiogr. 2007;20:1236-42.

3. Maffessanti F, Gripari P, Pontone G, Andreini D, Bertella E, Mushtaq S, et al. Three-dimensional dynamic assessment of tricuspid and mitral annuli using cardiovascular magnetic resonance. Eur Heart J Cardiovasc Imaging. 2013;14: 986-95.

4. Kabasawa M, Kohno H, Ishizaka T, Ishida K, Funabashi N, Kataoka A, et al. Assessment of functional tricuspid regurgitation using 320-detectorrow multislice computed tomography: risk factor analysis for recurrent regurgitation after tricuspid annuloplasty. J Thorac Cardiovasc Surg. 2014; $147: 312-20$

5. Van Rosendael PJ, Kamperidis V, Kong WKF, Van Rosendael AR, Van Der Kley F, Marsan NA, et al. Computed tomography for planning transcatheter tricuspid valve therapy. Eur Heart J. 2017;38:665-74.

6. Tei C, Pilgrim JP, Shah PM, Ormiston JA, Wong M. The tricuspid valve annulus: study of size and motion in normal subjects and in patients with tricuspid regurgitation. Circulation. 1982;66:665-72.

7. Fukuda S, Saracino G, Matsumura Y, Daimon M, Tran H, Greenberg NL, et al. Three-dimensional geometry of the tricuspid annulus in healthy subjects and in patients with functional tricuspid regurgitation: a real-time, 3-dimensional echocardiographic study. Circulation. 2006;114(Suppl 1):I492-8.

8. Kwan J, Kim GC, Jeon MJ, Kim DH, Shiota T, Thomas JD, et al. 3D geometry of a normal tricuspid annulus during systole: a comparison study with the mitral annulus using real-time 3D echocardiography. Eur J Echocardiogr. 2007;8: 375-83.

9. Addetia K, Muraru D, Veronesi F, Jenei C, Cavalli G, Besser SA, et al. 3-Dimensional echocardiographic analysis of the tricuspid annulus provides new insights into tricuspid valve geometry and dynamics. JACC Cardiovasc Imaging. November 10, 2017 [Epub ahead of print].

10. Dreyfus GD, Martin RP, Chan KMJ, Dulguerov F, Alexandrescu C. Functional tricuspid regurgitation: a need to revise our understanding. J Am Coll Cardiol. 2015;65:2331-6.

11. Gorman JHI, Gupta KB, Streicher JT, Gorman RC, Jackson BM, Ratcliffe MB, et al. Dynamic three-dimensional imaging of the mitral valve and left ventricle by rapid sonomicrometry array localization. J Thorac Cardiovasc Surg. 1996;112: 712-26.

12. Hiro ME, Jouan J, Pagel MR, Lansac E, Lim KH, Lim H-S, et al. Sonometric study of the normal tricuspid valve annulus in sheep. J Heart Valve Dis. 2004; 13:452-60.

13. Fawzy H, Fukamachi K, Mazer CD, Harrington A, Latter D, Bonneau D, et al. Complete mapping of the tricuspid valve apparatus using three-dimensional sonomicrometry. J Thorac Cardiovasc Surg. 2011;141:1037-43.

14. Malinowski M, Wilton P, Khaghani A, Langholz D, Hooker V, Eberhart L, et al. The effect of pulmonary hypertension on ovine tricuspid annular dynamics. Eur J Cardiothoracic Surg. 2016;49:40-5.

15. Ardehali A, Esmailian F, Deng M, Soltesz E, Hsich E, Naka Y, et al. Ex-vivo perfusion of donor hearts for human heart transplantation (PROCEED II): a prospective, open-label, multicentre, randomised noninferiority trial. Lancet. 2016;385:2577-84.

16. Rausch MK, Malinowski M, Wilton P, Khaghani A, Timek TA. Engineering analysis of tricuspid annular dynamics in the beating ovine heart. Ann Biomed Eng. 2018;46:443-51.

17. Rausch MK, Bothe W, Kvitting JPE, Swanson JC, Ingels NB, Miller DC. Characterization of mitral valve annular dynamics in the beating heart. Ann Biomed Eng. 2011;39:1690-702.

18. Silver MD, Lam JHC, Ranganathan N, Wigle ED. Morphology of the human tricuspid valve. Circulation. 1971;43:333-48.

19. Owais K, Taylor CE, Jiang L, Khabbaz KR, Montealegre-Gallegos M, Matyal R, et al. Tricuspid annulus: a three-dimensional deconstruction and reconstruction. Ann Thorac Surg. 2014;98:1536-42.

20. Leng S, Jiang M, Zhao XD, Allen JC, Kassab GS, Ouyang RZ, et al. Threedimensional tricuspid annular motion analysis from cardiac magnetic resonance feature-tracking. Ann Biomed Eng. 2016;44:3522-38.

21. Salgo IS, Gorman JH, Gorman RC, Jackson BM, Bowen FW, Plappert T, et al. Effect of annular shape on leaflet curvature in reducing mitral leaflet stress. Circulation. 2002;106:711-7.

22. Jimenez JH, Liou SW, Padala M, He Z, Sacks M, Gorman RC. A saddle-shaped annulus reduces systolic strain on the central region of the mitral valve anterior leaflet. J Thorac Cardiovasc Surg. 2007;134:1562-8.

23. Spinner EM, Buice D, Yap CH, Yoganathan AP. The effects of a three-dimensional, saddle-shaped annulus on anterior and posterior leaflet 
stretch and regurgitation of the tricuspid valve. Ann Biomed Eng. 2012;40: 996-1005.

24. Tsakiris AG, Mair DD, Seki S, Titus JL, Wood EH. Motion of the tricuspid valve annulus in anesthetized intact dogs. Circ Res. 1975;36:43-8.

25. Yamaura Y, Yoshikawa J, Yoshida K, Hozumi T, Akasaka T, Okada Y. Three-dimensional analysis of configuration and dynamics in patients with an annuloplasty ring by multiplane transesophageal echocardiography: comparison between flexible and rigid annuloplasty rings. $J$ Heart Valve Dis. 1995;4:618-22.

26. Glasson JR, Komeda M, Daughters GT, Foppiano LE, Bolger AF, Tye TL, et al. Most ovine mitral annular three-dimensional size reduction occurs before ventricular systole and is abolished with ventricular pacing. Circulation. 1997; 96(9 Suppl):II115-22; discussion II-123.

27. Basu A, Lacerda C, He Z. Mechanical properties and composition of the basal leaflet-annulus region of the tricuspid valve. Cardiovasc Eng Technol. 2018;9: 217-25.
28. Malinowski M, Wilton P, Khaghani A, Brown M, Langholz D, Hooker V, et al The effect of acute mechanical left ventricular unloading on ovine tricuspid annular size and geometry. Interact Cardiovasc Thorac Surg. 2016;23:391-6.

29. Medtronic. Healthcare professionals: Contour 3D Annuloplasty ring. Available at: http://www.medtronic.com/us-en/healthcare-professionals/products/cardiova scular/heart-valves-surgical/contour-3d.html. Accessed October 1, 2018.

30. McCarthy PM, Rhee RS, Schreck SG, inventors; Edwards Lifesciences Corp, assignee. Conformal tricuspd annuloplasty ring and template. US patent 7367 991. May 6, 2008

31. Navia JL, Nowicki ER, Blackstone EH, Brozzi NA, Nento DE, Atik FA, et al. Surgical management of secondary tricuspid valve regurgitation: annulus, commissure, or leaflet procedure? J Thorac Cardiovasc Surg. 2010;139:1473-82.e5.

Key Words: tricuspid valve, tricuspid annulus, strain, sonomicrometry 
TABLE E1. Raw hemodynamic data during baseline conditions showing stability of 3 heart cycles

\begin{tabular}{|c|c|c|c|c|c|c|c|c|}
\hline \multirow[b]{2}{*}{ Heart } & \multicolumn{2}{|c|}{ HR (bpm) } & \multicolumn{3}{|c|}{ RAP (mm Hg) } & \multicolumn{3}{|c|}{ RVP (mm Hg) } \\
\hline & Beat 1-2 & $\overline{\text { Beat 2-3 }}$ & $\overline{\text { Beat } 1}$ & Beat 2 & $\overline{\text { Beat } 3}$ & Beat 1 & Beat 2 & Beat 3 \\
\hline 1 & 80.3247 & 80.5133 & 4.4271 & 4.4323 & 4.5219 & 26.5125 & 26.5125 & 26.7969 \\
\hline 2 & 79.6572 & 79.6572 & 5.1004 & 5.1482 & 5.22 & 26.4604 & 26.4604 & 26.5552 \\
\hline 3 & 74.2436 & 74.2436 & 5.98 & 5.8978 & 5.9559 & 31.6237 & 31.7181 & 31.7181 \\
\hline 4 & 80.4957 & 79.6572 & 6.2297 & 6.2817 & 6.3019 & 20.7181 & 20.8124 & 20.9067 \\
\hline 5 & 80.4957 & 78.836 & 12.7974 & 12.5998 & 12.5316 & 30.2271 & 30.2271 & 29.9441 \\
\hline 6 & 79.6572 & 79.6572 & 3.0184 & 2.9466 & 2.9945 & 31.8442 & 31.8442 & 31.7494 \\
\hline 7 & 79.6572 & 80.4957 & 6.6095 & 6.6095 & 6.3931 & 27.5106 & 27.5106 & 27.7936 \\
\hline 8 & 80.4957 & 80.4957 & 4.68 & 4.6228 & 4.536 & 28.4163 & 28.4163 & 28.4163 \\
\hline 9 & 80.6572 & 80.4957 & 3.342 & 3.342 & 3.2788 & 28.3785 & 29.0389 & 28.3785 \\
\hline 10 & 80.6572 & 79.6572 & 4.2528 & 4.067 & 4.0789 & 35.2083 & 35.4913 & 35.4913 \\
\hline 11 & 79.6572 & 80.0339 & 7.0953 & 7.0953 & 6.5665 & 24.7933 & 24.7933 & 25.1707 \\
\hline
\end{tabular}

$H R$, Heart rate; $R A P$, right atrial pressure; $R V P$, right ventricular pressure.

TABLE E2. Hemodynamic parameters after calcium administration $(\mathbf{n}=\mathbf{5})$

\begin{tabular}{lccc}
\hline \multicolumn{1}{c}{ Parameter } & Precalcium & Calcium & $P$ value \\
\hline Heart rate $(\mathrm{bpm})$ & $80 \pm 1$ & $82 \pm 3$ & .353 \\
$\operatorname{RVP}_{\max }(\mathrm{mm} \mathrm{Hg})$ & $30 \pm 3$ & $36 \pm 14$ & .391 \\
RV EDP $(\mathrm{mm} \mathrm{Hg})$ & $8 \pm 4$ & $6 \pm 4$ & .115 \\
RV FAC $(\%)^{*}$ & $7 \pm 3$ & $9 \pm 4$ & .014 \\
RAP $(\mathrm{mm} \mathrm{Hg})$ & $6 \pm 2$ & $4 \pm 2$ & .095 \\
RV EDV $(\mathrm{mL})$ & $97 \pm 26$ & $80 \pm 14$ & .12 \\
\hline
\end{tabular}

Data are presented as mean \pm SD. $R V P$, Right ventricular pressure; $R V E D P$, right ventricular end diastolic pressure; $R V F A C$, right ventricular fractional area change, $R A P$, right atrial pressure; $R V E D V$, right ventricular end diastolic volume. *Data obtained from sonomicrometry. 